ESJ Humanities

\title{
Impacts De La Politique De Reconversion Des Systèmes D'irrigation Gravitaire vers l'irrigation Localisée : Cas Du Sous Bassin Du Tadla Azilal
}

\author{
Fatima Ezzahra Mengoub, Doctorante
}

Institut Agronomique et vétérinaire Hassan II, Rabat, Maroc

Caroline Lejars, Dr.

Centre de coopération internationale en recherche agronomique pour le développement, France

Mohammed Rachid Doukkali, Pr.

Institut Agronomique et vétérinaire Hassan II, Rabat, Maroc

Doi:10.19044/esj.2021.v17n24p46

Submitted: 15 June 2021

Accepted: 05 July 2021

Published: 31 July 2021
Copyright 2021 Author(s)

Under Creative Commons BY-NC-ND

4.0 OPEN ACCESS

Cite As:

Ezzahra Mengoub F., Lejars C. \& Rachid Doukkali M. (2021). Impacts De La Politique De Reconversion Des Systèmes D'irrigation Gravitaire vers l'irrigation Localisée : Cas Du Sous Bassin Du Tadla Azilal. European Scientific Journal, ESJ, 17(24), 46.

https://doi.org/10.19044/esj.2021.v17n24p46

\section{Résumé}

Au Maroc, les ressources en eau se font de plus en plus rares. De plus, celles-ci subissent plusieurs pressions notamment l'augmentation de leur demande ainsi que les effets négatifs du changement climatique. Devant cette situation, le gouvernement marocain a multiplié les efforts en termes d'intervention et de politique, que ce soit au niveau technique, juridique et/ou institutionnel. Adopté en 2009, le dernier programme national d'économie d'eau et d'irrigation envisage de reconvertir des systèmes d'irrigations traditionnels vers l'irrigation localisée. Ce travail vise l'évaluation des impacts économiques et sur les ressources en eau ce programme dans le sous basin de Tadla-Azilal. L'application d'un modèle de gestion intégrée montre qu'après reconversion, la marge brute agricole totale dans la zone augmenterait de $2 \%$. Des changements s'effectueront aux niveaux des assolements, notamment le remplacement de certaines cultures par d'autres, devenues beaucoup plus rentables. L'application des volumes d'eau précis aux moments des pics de demande des plantes se traduira par une augmentation des rendements de toutes les cultures. Quant aux consommations en eau, celles-ci baisseront 
significativement au niveau de toutes les unités étudiées et les nappes souterraines seront moins sollicitées qu'avant.

Mots clés : Maroc, Irrigation, Gestion Intégrée De L'eau

\title{
Economic and Resource Impacts of the Policy of Conversion to Localized Irrigation Systems: Case of the Tadla Azilal Region
}

Fatima Ezzahra Mengoub, Doctorante

Institut Agronomique et vétérinaire Hassan II, Rabat, Maroc

Caroline Lejars, Dr.

Centre de coopération internationale en recherche agronomique pour le développement, France

Mohammed Rachid Doukkali, Pr.

Institut Agronomique et vétérinaire Hassan II, Rabat, Maroc

\begin{abstract}
In Morocco, water resources are becoming increasingly scarce. In addition, they are facing several pressures, including increased demand and the negative effects of climate change. In response to this situation, the Moroccan government has increased its efforts in terms of intervention and policy, whether at the technical, legal and/or institutional level. Adopted in 2009 , the latest national water saving and irrigation program envisages the conversion of traditional irrigation systems to localized irrigation. This work aims at evaluating the economic and water resources impacts of this program in the Tadla-Azilal sub-basin. The application of an integrated management model shows that after reconversion, the total agricultural gross margin in the area would increase by $2 \%$. Changes will be made in crop rotation, including the replacement of certain crops by others that have become much more profitable. The application of specific water volumes at times of peak plant demand will result in increased yields of all crops. Finally, water consumption will be significantly reduced in all the studied units and groundwater will be less solicited than before.
\end{abstract}

Keywords: Morocco, Irrigation, Integrated Water Management

\section{Introduction}

$\mathrm{Au}$ Maroc, la pression sur les ressources en eau s'accroit du fait de plusieurs facteurs. Ces derniers sont de l'ordre climatique se traduisant par des 
sécheresses récurrentes et la baisse des niveaux de précipitation; démographique vu que la population connait une croissance significative ; et économique en raison des exigences du développement des secteurs agricole, touristique et industriel (Boukhari et al., 2019). Face à cette situation, l'Etat marocain a multiplié les efforts aussi bien sur le plan technique, qu'au niveau juridique et institutionnel pour atténuer l'impact des aléas climatiques et mieux gérer les ressources hydriques (Global Water Partnership, 2012; Benouniche, 2014 ; Bennouna, 2020). A travers ses diverses politiques, le gouvernement marocain, a d'abord investi des montants colossaux pour développer un patrimoine hydrique important. On estime à environ 20 milliards de dirhams par an le montant global débloqué par les pouvoirs publics pour développer l'infrastructure de base (Molle et al., 2019). Soixante pourcent de ce montant ont été destinés au secteur de l'eau potable et l'assainissement, vingt pourcent ont permis de promouvoir l'irrigation alors que vingt pourcent ont été dépensés pour transférer l'eau des zones les plus arrosées aux zones sèches (CESE, 2014). Ensuite, le souci d'amélioration de l'efficience de l'eau et de la durabilité de son utilisation a incité les pouvoirs publics à adopter des instruments de politiques visant à rationaliser son utilisation (Belghiti, 2004). La tarification de l'eau d'irrigation, proposée dans le cadre du code d'investissement agricole (CIA, 1969), est l'un des instruments majeurs mis en place pour remédier aux différentes inefficiences (Serghini, 2003).

Récemment, la raréfaction de l'eau au Maroc a incité le gouvernement à repenser sa stratégie de l'eau et à mettre en œuvre de nouvelles mesures pour mieux gérer ces ressources. Ainsi, le gouvernement a mis en place plusieurs programmes de gestion de l'eau dont le Plan National d'Economie d'Eau d'Irrigation (PNEEI). Ce dernier vise la modernisation d'une bonne partie des réseaux collectifs d'irrigation et la reconversion des systèmes d'irrigation existants vers l'irrigation localisée. L'objectif est d'équiper près de 550000 ha situés dans les périmètres d'irrigation collectifs et privés en irrigation localisée (Arrifi, 2009).

A lumière de ce qui précède, ce travail vise à évaluer l'impact de la reconversion des systèmes d'irrigation existants vers l'irrigation localisée. Ainsi il a pour but d'évaluer l'impact de l'introduction d'une nouvelle technique d'irrigation sur les revenus des agriculteurs, la consommation en eau, la structure des assolements pratiqués et enfin le prix économique de l'eau.

\section{Méthode d'approche : Conception d'un modèle de bassin versant}

Le modèle de gestion intégrée de bassin, élaboré pour cette étude, se base sur la simulation des flux d'eau, les équations d'équilibre des ressourcesemplois des retenues d'eau et les flux d'eau au niveau des unités 
hydrologiques. La structure générale du modèle se base sur trois composantes : l'hydrologie, l'agronomie et l'économie (Cai, 1999 ; Cai et al., 2006 ; Heidecke et al., 2006). Ces trois composantes sont intégrées de façon à reprendre les règles d'allocation de l'eau, le fonctionnement hydrologique des différents systèmes et les différentes demandes en eau dans la zone d'étude. En confrontant l'offre à la demande, ce modèle permet également de calculer le prix économique ou "Shadow price » de l'eau dans les différents souspérimètres étudiés. Défini dans notre cas comme l'augmentation marginale de la marge brute résultante de l'utilisation supplémentaire d'une unité d'eau, ce prix reflète le degré de rareté de l'eau (Tsur et al., 2004). Ainsi, il permet d'évaluer l'impact de l'introduction d'une nouvelle technologie d'irrigation, jugée plus économe, sur le degré de rareté de l'eau dans la zone étudiée.

\section{a) Composante hydrologique}

Le module hydrologique est constitué d'un ensemble d'équations décrivant l'ensemble des flux entrants et sortants au niveau du bassin et au niveau de chaque unité de demande au sein du bassin. Les flux entrants au niveau de chaque unité de demande sont constitués des apports des différentes sources : retenues d'eau, prélèvements des eaux de sources, prélèvement à partir des nappes souterraines et précipitations. Les flux sortants du bassin sont constitués de l'évapotranspiration, infiltration, retour d'irrigation, drainage et écoulement vers la rivière.

\section{Equilibre au niveau des retenues d'eau (Barrages/eau superficielle)}

Le bilan hydrique au niveau des retenues d'eau est calculé en prenant en considération tous les flux entrant et sortant des barrages (Vincing et al. 2007 ; Güntner et al., 2009 ; Stella, 2019). Pour cette étude, le stock de l'eau au niveau de la retenue $(\mathrm{R})$ en fin de période $\mathrm{t}\left(\mathrm{Stck}_{\mathrm{R}, \mathrm{t}}\right)$ est égal au stock en fin de période $\mathrm{t}-1$ (Stck $\left.\mathrm{R}_{\mathrm{R}, \mathrm{t}-1}\right)$, plus les apports résultant des écoulements d'eau et de la fonte des neiges à l'amont de la retenue $\left(\mathrm{AP}_{\mathrm{R}, \mathrm{t}}\right)$, moins les différentes sorties : dotations en eau potable $\left(\mathrm{EP}_{\mathrm{R}, \mathrm{t}}\right)$, dotations en eau d'irrigation $\left(\mathrm{EI}_{\mathrm{R}, \mathrm{t}}\right)$, turbinage $\left(\mathrm{ET}_{\mathrm{R}, \mathrm{t}}\right)$, transferts interbassin $\left(\mathrm{TIB}_{\mathrm{R}, \mathrm{t}}\right)$ et évaporation au niveau de la retenue $\left(\mathrm{EVAP}_{\mathrm{R}, \mathrm{t}}\right)$. 


$$
\operatorname{Stck}_{\mathrm{R}, \mathrm{t}}=\mathrm{Stck}_{\mathrm{R}, \mathrm{t}-1}+\mathrm{AP}_{\mathrm{R}, \mathrm{t}}-\mathrm{EP}_{\mathrm{R}, \mathrm{t}}-\mathrm{EI}_{\mathrm{R}, \mathrm{t}}-\mathrm{ET}_{\mathrm{R}, \mathrm{t}}-\mathrm{TIB}_{\mathrm{R}, \mathrm{t}}-\mathrm{EVAP}_{\mathrm{R}, \mathrm{t}}
$$

L'ensemble des variables entrant dans cette équation sont déterminées de façon exogène, seule la dotation en eau d'irrigation est calculée de façon endogène. Cette dernière dépend de plusieurs facteurs : la pluviosité annuelle, disponibilité d'eau dans le réservoir (selon les règles de gestion des eaux des barrages adoptés par les autorités), des cultures pratiquées (décision de l'agriculteur), la possibilité de pompage et le coût de pompage (en cas de pratique de l'irrigation conjointe).

\section{Equilibre au niveau des différents nouds à l'amont de l'exutoire}

Au niveau de chaque nœud à l'amont de l'exutoire, l'équilibre entre les flux entrants et les flux sortants doit être respecté. Selon le nœud en question, les flux entrants peuvent être constitués des apports en amont du nœud [dotation des eaux du barrage $\left(\operatorname{Dot}_{\mathrm{R}, \mathrm{n}, \mathrm{t}}\right)$ ou prélèvement directement dans la rivière $\left(\operatorname{RivN}_{\mathrm{n}, \mathrm{t}}\right)$ ], les entrées latérales $\left(\right.$ EntLat $\left._{\mathrm{n}, \mathrm{t}}\right)$ ou la décharge de la nappe vers le nœud en question $\left(\operatorname{DechN}_{\text {nap,n,t }}\right)$. Les flux sortants peuvent avoir trois destinations soit vers un réservoir $\left(F_{l u N R}, \mathrm{R}, \mathrm{t}\right)$, soit vers une unité de demande d'eau d'irrigation $\left(\right.$ FluND $\left._{\mathrm{n}, \mathrm{d}, \mathrm{t}}\right)$ soit vers la rivière $\left(\mathrm{NRiv}_{\mathrm{n}, \mathrm{t}}\right)$.

$$
\begin{array}{r}
\operatorname{DotR}_{\mathrm{R}, \mathrm{n}, \mathrm{t}}+\operatorname{RivN}_{\mathrm{n}, \mathrm{t}}+\text { EntLat }_{\mathrm{n}, \mathrm{t}}+\text { DechN }_{\mathrm{nap}, \mathrm{n}, \mathrm{t}}=\text { FluNR }_{\mathrm{n}, \mathrm{R}, \mathrm{t}}+\text { FluND }_{\mathrm{n}, \mathrm{d}, \mathrm{t}}+ \\
\operatorname{NRiv}_{\mathrm{n}, \mathrm{t}}
\end{array}
$$

\section{Equilibre au niveau des nappes}

La quantité d'eau extraite des nappes doit être en fonction de tous du stock d'eau au niveau de la nappe, des flux entrants (précipitations et retour d'eau d'irrigation) et enfin la décharge de la nappe (Zargham et al. ; Dhungel et al., 2016). Ainsi, la variation des réserves en eau au niveau de la nappe (nap) par période $t\left(D E N_{n a p, t}\right)$ est le produit de la hauteur piézométrique $\left(Z_{n a p, t}\right)$ par le coefficient d'emmagasinement de la nappe $\left(\mathrm{CMG}_{\text {nap }}\right)$ et par la superficie de la nappe $\left(\mathrm{SNAP}_{\text {nap }}\right)$, soit :

$$
\mathrm{DEN}_{\text {nap }, \mathrm{t}}=\mathrm{Z}_{\text {nap,t }} \cdot \mathrm{CMG}_{\text {nap }} \cdot \mathrm{SNAP}_{\text {nap }}
$$

Pour calculer cette variation, il est nécessaire d'établir l'équation d'équilibre entre les entrées et les sorties de la nappe, qui se calcule comme suit :

$$
\begin{aligned}
& \left(\operatorname{RechPl}_{\text {nap,t }}\right)_{+}\left(\text {FLuLatE }_{\text {nap, }}\right)+\left(\operatorname{InfUD}_{\mathrm{d}, \text { nap }, \mathrm{t}}\right)+\left(\operatorname{InfUD}_{\mathrm{d}, \mathrm{nap}, \mathrm{t}}\right)+
\end{aligned}
$$

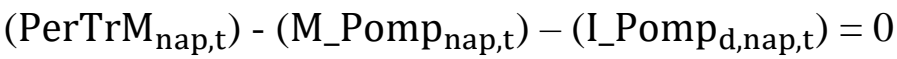


Où les variables sont :

- la recharge naturelle de la nappe par les eaux de pluie par période $t$ $\left(\operatorname{RechPl}_{\text {nap,t }}\right)$

- les flux latéraux ou verticaux entrants à partir d'autres nappes pendant la période $\mathrm{t}\left(\mathrm{FLuLatE}_{\mathrm{nap}, \mathrm{t}}=\sum_{\text {napp } \neq \text { nap }}\right.$ FLuLat $\left._{\text {napp,t }}\right)$,

- La somme des percolations profondes des unités de demande (d) pendant la période $t\left(\operatorname{InfUD}_{\mathrm{d}, \mathrm{nap}, \mathrm{t}}\right)$,

- Les infiltrations dues aux pertes des eaux d'irrigation dans les réseaux de transport $\left(\operatorname{PerTrI}_{\mathrm{d}, \mathrm{nap}, \mathrm{t}}\right)$,

- Les infiltrations des pertes des eaux municipales dans les réseaux de transport (PerTrM $\left.{ }_{\text {nap,t }}\right)$,

- Les prélèvements de l'eau municipale dans la nappe (M_Pomp nap,t $\left._{-}\right)$, moins les prélèvements des eaux d'irrigation par les unités de demande (I_Pomp ${ }_{d, n a p, t}$ ),

- Les flux latéraux sortant vers les autres nappes $\left(\right.$ FLuLatS $_{\text {nap,t }}=$ $\sum_{\text {napp } \neq \text { nap }}$ FLuLat $_{\text {napp,t }}$

- moins la décharge vers la rivière ou exutoire $\left(\operatorname{DechR}_{\text {nap,t }}\right)$

\section{Équations des utilisations de l'eau pour l'irrigation}

Les unités de demande en eau reçoivent de l'eau à partir des barrages $\mathrm{EI}_{\mathrm{R}, \mathrm{t}}$. Sachant qu'une partie de cette quantité d'eau est perdue au niveau du réseau d'adduction de l'eau, la quantité d'eau d'irrigation effective reçue par les unités de demande peut s'écrire de la manière suivante :

$$
\sum_{\mathrm{R}} \mathrm{EIR}_{\mathrm{R}, \mathrm{t}}=\mathrm{EI}_{\mathrm{R}, \mathrm{t}}-\sum_{\mathrm{d}} \operatorname{PerTrI}_{\mathrm{d}, \mathrm{t}}=\mathrm{EI}_{\mathrm{R}, \mathrm{t}} \cdot(1-\text { CoefPrTran })
$$

CoefPrTran : Efficience des réseaux de transport : coefficient des pertes au niveau des réseaux du transport de l'eau d'irrigation

Les quantités d'eau d'irrigation appliquées ont principalement deux sources (eau superficielle ou souterraine). Ainsi, la quantité totale d'eau d'irrigation utilisée par les agriculteurs $\left(\sum_{\mathrm{d}} \mathrm{EIR}_{\mathrm{d}}\right)$ sera dans ce modèle la somme des quantités d'eaux superficielles reçues à partir des barrages $\left(\sum_{\mathrm{R}} \mathrm{EIR}_{\mathrm{R}, \mathrm{t}}\right)$ et les volumes d'eau pompés à partir des nappes $\left(\sum_{\mathrm{d}} \mathrm{I}_{\mathrm{Pomp}} \mathrm{d}_{\mathrm{d}, \mathrm{nap}, \mathrm{t}}\right)$

$$
\sum_{\mathrm{d}} \mathrm{EIR}_{\mathrm{d}}=\sum_{\mathrm{R}} \mathrm{EIR}_{\mathrm{R}, \mathrm{t}}+\sum_{\mathrm{d}} \mathrm{I}_{\text {Pomp }_{\mathrm{d}, \text { nap }, \mathrm{t}}}
$$

\section{a) La composante agronomique}


Au niveau de cette composante, il est question de calculer les rendements réalisés par cultures et par site de demande en eau. La variable clé est les quantités d'eau d'irrigation appliquées qui, confrontées aux besoins des cultures, détermineront les niveaux de production atteint dans la zone d'étude.

Pour calculer la quantité d'eau appliquée par culture selon les sites de demande, il faut respecter l'égalité suivante :

$$
\mathrm{EI} / \mathrm{Ha}_{\mathrm{d}, \text { cult }, \mathrm{t}}+\mathrm{PEff}_{\mathrm{d}, \mathrm{t}} \cdot 0.01=\mathrm{ETA}_{\mathrm{d}, \text { cult }, \mathrm{t}}+\operatorname{InfIRC}_{\mathrm{d}, \mathrm{cult}, \mathrm{t}}
$$

Où :

$\mathrm{EI} / \mathrm{Ha}_{\mathrm{d}, \mathrm{cult}, \mathrm{t}} \quad$ : La quantité d'eau d'irrigation appliquée par hectare selon les cultures et les sites de demande d'eau

PEff $_{\mathrm{d}, \mathrm{t}} \quad$ : La pluie efficace par site de demande et par mois

ETA $_{\mathrm{d}, \text { cult,t }} \quad$ : Évapotranspiration actuelle des cultures par mois selon les sites de demande.

InfIRC $_{\mathrm{d}, \text { cult,t }} \quad$ : L'infiltration de l'eau d'irrigation par période selon les cultures pratiquées et le site de demande.

Basé sur le modèle développé par Cai (1999), le calcul des infiltrations de l'eau au niveau des parcelles est en fonction de l'efficience de l'irrigation $\left(\operatorname{effIR}_{\mathrm{d}, \mathrm{cult}, \mathrm{t}}\right)$. Il se base sur l'équation suivante :

$$
\operatorname{InfIRC}_{\mathrm{d}, \text { cult }, \mathrm{t}}=\left(\mathrm{EI} / \mathrm{Ha}_{\mathrm{d}, \text { cult }, \mathrm{t}}+\mathrm{PEff}_{\text {nap }, \mathrm{t}} \cdot 0.01\right) \cdot \text { effIR }_{\mathrm{d}, \text { cult }, \mathrm{t}}
$$

En ce qui concerne l'évapotranspiration actuelle des cultures par mois selon les sites de demande $\left(\mathrm{ETA}_{\mathrm{d}, \text { cult }, \mathrm{t}}\right)$, le modèle considère les équations développées par Hanks (1974). Dans ce travail, elle doit être égale aux évapotranspirations des cultures par site de demande $\left(\mathrm{ETAD}_{\mathrm{d}, \text { cult }}\right)$ multipliées par les besoins mensuels maximums $\left(\mathrm{ETP}_{\mathrm{t}, \text { cult }}\right)$ rapportés à la somme des besoins en eau maximums $\left(\sum_{\mathrm{t}} \mathrm{ETP}_{\mathrm{t}, \text { cult }}\right)$.

$$
\mathrm{ETA}_{\mathrm{d}, \mathrm{cult}, \mathrm{t}}=\mathrm{ETAD}_{\mathrm{d}, \text { cult }} \cdot \frac{\mathrm{ETP}_{\mathrm{t}, \text { cult }}}{\sum_{\mathrm{t}} \mathrm{ETP}_{\mathrm{t}, \text { cult }}}
$$

Enfin, le rendement $\left(\mathrm{RDMT}_{\mathrm{a}}\right)$ se calcule à partir de l'équation de FAO (2012), il s'agit de la fonction de réponse de rendement à l'eau qui tient compte du déficit en eau (ETA/ETP), du coefficient de réponse $\left(\mathrm{K}_{\mathrm{y}}\right)$ et du rendement maximal (RDMT_Max cult $_{\text {) }}$ 


$$
\left(1-\frac{\text { RDMT }_{\text {cult }}}{\text { RDMT_Max }_{\text {,cult }}}\right)=K_{y}\left(1-\frac{\text { ETA }_{\text {d,cult }}}{\text { ETP }_{\text {d,cult }}}\right)
$$

\section{b) La composante économique}

Une fois les variables techniques sont déterminées, le modèle maximise la marge brute agricole totale produite au niveau de la zone d'étude en s'appuyant sur la fonction objectif suivante :

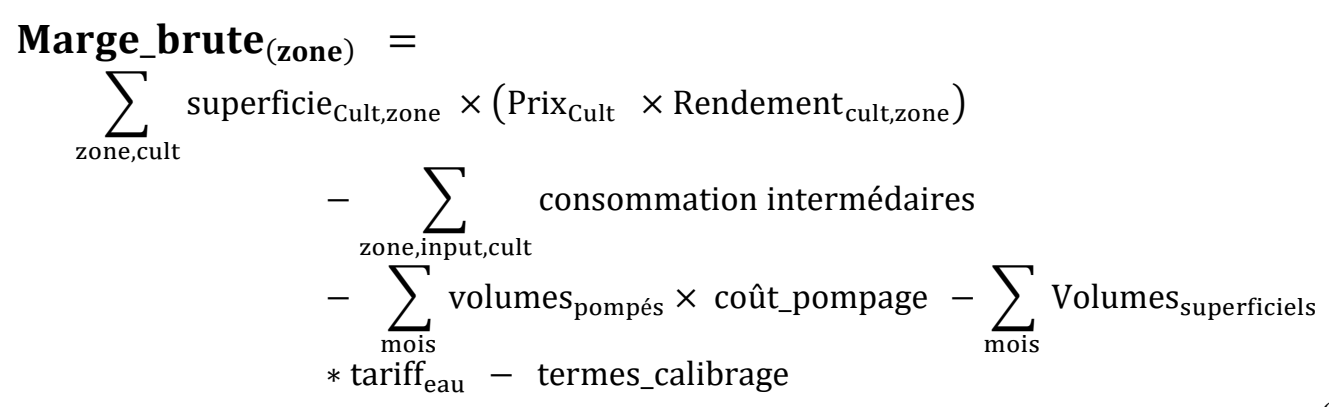

Pour chaque site de demande, l'équation ci-dessus calcule la marge brute des cultures, c'est-à-dire les superficies allouées aux cultures multipliée par leurs rendements et leurs prix de vente unitaires, moins toutes les charges variables, y compris celles liées à l'irrigation. Cette marge brute est maximisée, compte tenu des différentes contraintes, à savoir :

1. La contrainte terre traduisant le fait que les agriculteurs ont une superficie physique limitée pour produire.

2. La contrainte liée à la disponibilité de l'eau d'irrigation. Les quantités d'eau disponibles calculées par les équations d'équilibre des entrées et sorties d'eau au niveau des retenues d'eau et des nappes souterraines.

3. Le groupe de contraintes agronomiques regroupant tous les paramètres techniques et agronomiques jouant un rôle capital dans la formation des rendements.

\section{Aire de l'étude et spécification du modèle}

L'étude a porté sur le sous bassin de Tadla-Azilal, l'une des zones agricoles les plus importantes au Maroc. La démarche est de conduire la simulation d'impact, basée sur l'élaboration d'un modèle de gestion intégrée des ressources en eau. Il s'agit d'un outil d'aide à la décision qui tient compte des différents usagers et qui permet d'évaluer l'effet d'une politique d'économie d'eau sur les éléments clés du développement agricole (Global Water Partnership, 2009). Pour cette étude, les variables clés sont la marge 
brute agricole générée au niveau de l'unité d'étude et ventilée selon la source de l'eau d'irrigation, les rendements des cultures, les quantités des eaux de surface utilisées ainsi que celles des eaux souterraines. Le modèle est désagrégé selon les différents sites de demande en eau $\left(\mathrm{GH}^{1}, \mathrm{PMH}^{2}, \mathrm{IP}^{3}\right.$ et Bour ${ }^{4}$ ), les sources d'eau (superficielle et souterraine) et les cultures. L'évaluation s'effectuera en comparant la situation avant et après projet de reconversion du système d'irrigation vers l'irrigation localisée en année de pluviosité moyenne.

Pour mieux cerner et représenter la diversité des différents systèmes de production, le modèle retient les éléments suivants :

- Cinq sites de demande, où les exploitations agricoles sont différenciées selon la source de l'eau d'irrigation. Ces sites comprennent :

- Deux périmètres irrigués de grandes hydrauliques (périmètres des Béni Moussa et des Béni Amir). A l'intérieur de ces deux périmètres, deux types d'exploitations agricoles doivent être distingués. Le premier groupe est formé des agriculteurs irriguant leurs parcelles exclusivement à partir des eaux superficielles issues des deux barrages Ahmed El Hansali et de Bin El Ouidane. Le second groupe est formé des agriculteurs pratiquant l'irrigation complémentaire à partir des deux nappes phréatiques des Béni Amir et des Béni Moussa, en plus de l'irrigation à partir des eaux issues des mêmes deux barrages.

- Un périmètre de petite et moyenne hydraulique où figurent deux types d'exploitations agricoles: celles qui irriguent exclusivement à partir des sources d'eau et celle qui pratique l'irrigation conjointe.

- Un périmètre d'irrigation privée où les agriculteurs irriguent exclusivement à partir de la nappe profonde.

- Un périmètre consacré à l'agriculture pluviale où les exploitations agricoles ne sont pas irriguées et les besoins des plantes sont satisfaits à partir des eaux de pluie.

- Huit groupes de cultures qui sont les céréales, la betterave à sucre, les fourrages, les légumineuses, le maraichage, les agrumes, l'olivier et les autres plantations (grenadier, rosacées, etc.)

- Trois nappes phréatiques (nappes des Béni Moussa, des Béni Amir et celle du Dir)

- Une nappe profonde

- Deux grands barrages (Bin El Ouidane et Ahmed El Hansali).

${ }^{1} \mathrm{GH}$ : Grande Hydraulique

${ }^{2} \mathrm{PMH}$ : Petite et Moyenne hydraulique

${ }^{3}$ IP : Irrigation Privée à partir des eaux souterraines

${ }^{4}$ Bour : Agriculture Pluviale 
- Un canal soucier (canal du Dir)

\section{Les paramètres exogènes}

Représentés généralement par des paramètres de la production végétale ainsi que d'autres paramètres qui se présentent pour les différents secteurs.

- Paramètres agronomiques : les rendements potentiels par culture, les besoins en intrants, les superficies par cultures, la pluie effective, évapotranspiration maximale par culture et par stade de développement et coefficient de réponse du rendement par culture. Ces données ont été collecté à partir des enquêtes faites auprès des administrations publiques (offrice de mise en valeur agricole, direction régionale de l'agriculture, etc.)

- Paramètres non agronomiques : quantités consommées dans le secteur industriel et de l'eau potable collectés auprès de l'agence de bassin hydraulique. D'autres paramètre économique dont le prix de vente des produits agricoles, les prix des intrants, les redevances de l'eau d'irrigation et le coût de pompage selon la profondeur ont été collectés auprès des agriculteurs.

- Paramètres techniques et hydrologiques: efficience des réseaux de distribution pour l'eau agricole, industrielle et potable, évaporation au niveau des deux barrages, volume maximal de chaque nappe, coefficient de stockage, profondeur et perméabilité des nappes, les apports des barrages, les volumes régularisés des barrages et enfin les apports du canal soucier de la PMH. Ces paramètres ont été puisés des rapports techniques produits par l'agence de bassin hydraulique.

\section{Les paramètres endogènes}

Ce sont les différentes variables agronomiques, hydrologiques et économiques qui sont calculées par le modèle, notamment, les flux d'eau au niveau des nœuds du bassin, entrées et sorties des nappes, balance du barrage, demande en eau par culture, déficit hydrique des cultures, demande en eau d'irrigation par secteur agricole, les coûts marginaux de l'utilisation de l'eau et de la terre, etc. elles sont issues des équations d'équilibre du modèle. 


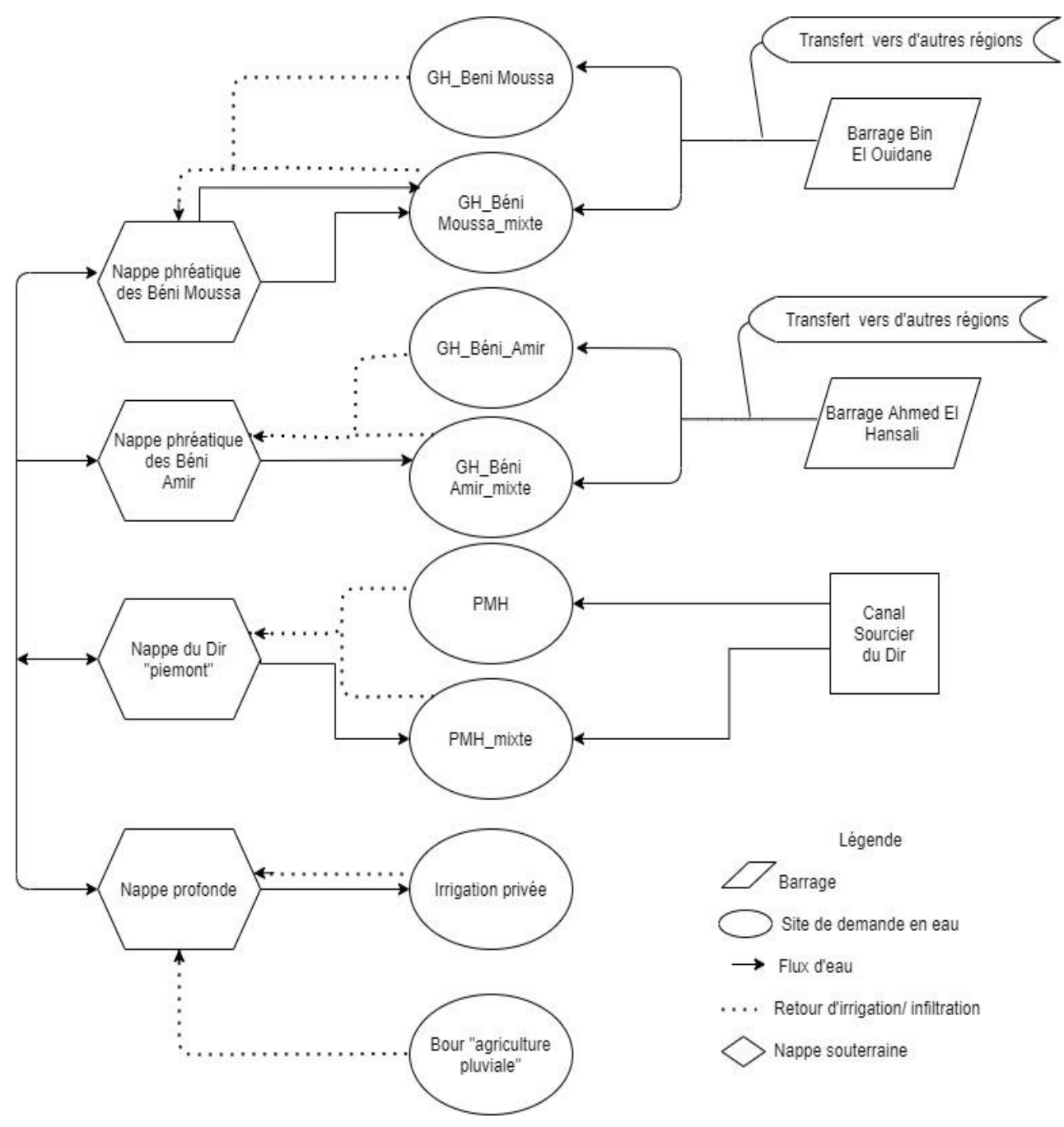

Figure 1 : représentation conceptuelle du réseau des nœuds, des sites de demandes et des retenues d'eau 


\section{Hypothèse du modèle}

Le modèle repose sur cinq hypothèses de base :

1- Le modèle calcule la valeur marginale économique ou le prix économique de l'eau pour les différents périmètres irrigués de la zone d'étude. Les ressources terre et eau d'irrigation sont utilisées de la manière optimale afin de maximiser le revenu au niveau des exploitations agrégées.

2- les demandes en eau municipales et industrielles sont introduites de façon exogène ${ }^{5}$ vu que ces derniers sont prioritaires et que l'agriculture est desservie en eau après avoir satisfait les demandes en eau municipale et industrielle.

3- Le pompage au niveau de la zone d'étude est illimité, ce qui signifie que les agriculteurs peuvent pomper l'eau sans contrainte limitante.

4- La surface totale au niveau du bassin est supposée entièrement utilisée.

5- Le rendement potentiel des cultures varie selon le système de production (irrigué vs pluvial) et les coûts de production dépendent des niveaux de production réalisés.

\section{Calibration du modèle}

La calibration de ce modèle est faite en s'appuyant sur la programmation mathématique positive (PMP) (Berger, 2010). La PMP est une technique de modélisation qui permet de contourner les principaux problèmes rencontrés en programmation mathématique classique, notamment la rigidité des modèles face aux variations des paramètres, la calibration ou l'absence de données (Howitt, 1995). La méthodologie de la PMP est basée sur l'idée qu'il est plus facile d'observer les niveaux de production des différents processus des entreprises que leurs coûts (Paris et al., 1996). La PMP permet de reproduire le choix des producteurs notamment les agriculteurs en estimant une fonction de coût. Cette dernière capte toutes charges, connues ou simplement perçues, ayant participé dans le processus de production. Une fois la situation de base reproduite, le modèle est prêt pour la phase suivante : la simulation.

D'une manière plus détaillée, la méthodologie de PMP s'articule en trois phases successives, chacune caractérisée par un objectif spécifique.

Phase 1 : Le calibrage de la situation de départ

La première étape consiste en la résolution d'un système linéaire où les contraintes se présentent sous la forme : $A . x \leq b$. L'objectif de cette étape est l'estimation des coûts implicites que les producteurs prennent en

${ }^{5}$ En prenant en considération que la demande en eau municipale et industrielle doit être satisfaite en premier lieu, ce modèle tient compte des valeurs réelles des quantités d'eau potable et industrielle consommées et les extrait des disponibilités en eau avant optimisation. 
considération lorsqu'ils définissent leur plan de production. Il s'agit des coûts marginaux différentiels qui doivent être ajoutés aux coûts comptables pour déterminer les coûts marginaux totaux associés aux différents processus de production.

Phase 2 : L'estimation de la matrice des coûts

L'étape suivante consiste en l'estimation de la fonction de coût non linéaire de type $A \cdot x^{2}+B \cdot x+C \leq b$ qui reprend les informations sur les coûts des différents processus de production et les relations de substitution et de complémentarité entre les différents produits.

Phase 3 : Le modèle de simulation

La fonction de coût, estimée dans la phase précédente, est incluse dans la formulation d'un nouveau problème de programmation mathématique semblable à celui de la première phase, mais qui comporte une composante de coût non linéaire dans la fonction objectif. Le nouveau modèle peut ainsi reproduire la situation de base sans que les contraintes des coûts implicites ne soient intégrées.

Dans cette étude, le paramètre du calibrage est la part dédiée à chaque culture dans la superficie agricole utile totale. Pour la phase 1, la part de chaque culture est fixée aux assolements observés dans chaque unité de demande. Les superficies réservées à chaque culture sont alors imposées dans le premier modèle et les résultats reprennent exactement les superficies dédiées à chaque culture et reproduisent les choix des agriculteurs. Pour la phase 2, la fonction objectif est modifiée dans le deuxième modèle. Elle intègre désormais un terme non linéaire de coûts implicites composé des coûts marginaux de l'utilisation de la terre. Cette nouvelle forme prend en considération les coûts des facteurs propres dont le prix est estimé à partir du premier modèle. Enfin pour la phase 3, une fois les termes de la fonction des coûts implicites calculés, la contrainte sur les assolements est libérée et le modèle maximise la marge brute par unité de demande.

\section{Année de référence et choix des simulations}

L'année de référence retenue pour cette étude correspond à la campagne agricole 2011-2012. Cette année de référence permettra de représenter la situation du secteur agricole pendant une année de pluviosité moyenne, dont le cumul annuel des pluies s'élève à $385 \mathrm{~mm}$. Elle a été obtenue en analysant une série de pluviométrie durant les 15 dernières années. En plus de représenter les caractéristiques hydro-climatiques d'une année moyenne, elle permettra également de comprendre les conflits d'usage autour de l'allocation de l'eau dans la zone d'étude et servira par la suite comme référence pour la comparaison des différents scénarios simulés. 
La simulation conduite dans cette étude consiste à évaluer l'impact de la reconversion du système d'irrigation gravitaire vers le système dit localisé sur la marge brute agricole totale pendant une année de pluviométrie moyenne. Dans le Tadla, le PNEEI vise à convertir environ 49000 ha se trouvant dans les périmètres d'irrigation collective et 39700 ha dans l'irrigation privée ou individuelle en dehors des périmètres irrigués de grande hydraulique (Boularbah et al., 2017). Dans ce sens, la simulation conduite suppose d'abord que la totalité des superficies conduites en irrigation privée sera reconvertie en goutte à goutte. Ensuite, que la majeure partie des superficies prévues pour être reconverties dans les périmètres d'irrigation collective s'effectuera au niveau du périmètre de grande hydraulique des Béni Moussa en raison de la qualité du sol, qui est nettement meilleure que celle des sols des Béni Amir (moins de salinité).

Du point de vue technique, il faut noter que le système d'irrigation goutte à goutte présente une efficience globale de l'ordre de $90 \%$ (Bouaziz et al., 2002), contrairement au système d'irrigation gravitaire dont l'efficience globale est d'environ $50 \%$. Nous faisons l'hypothèse que cette technique d'irrigation va réduire les pertes en eau pour les exploitations agricoles au niveau des conduites ainsi qu'au niveau des parcelles de $40 \%$ dans la zone d'étude. Avec l'installation du système d'irrigation localisée, des frais supplémentaires de filtrage et de pression s'ajouteront au niveau du coût de pompage de l'eau et aussi au niveau du coût de l'eau de surface. Le coût de pompage de l'eau souterraine et le coût de l'eau de surface augmenteront respectivement de $0,2 \mathrm{Dh} / \mathrm{m} 3$ et $0,15 \mathrm{Dh} / \mathrm{m} 3$, en raison des frais d'énergie qu'il faut prendre en considération pour effectuer des opérations liées à ce système d'irrigation, telles que le filtrage de l'eau et la régulation de sa pression (Mengoub et al., 2014).

\section{Résultats et discussion}

\section{- Impact de la reconversion des systèmes d'irrigation sur l'assolement}

Les résultats obtenus (Tableau 1) montrent qu'au niveau régional, l'introduction de l'irrigation localisée impacterait significativement les choix des agriculteurs. Des baisses des superficies allouées à cinq cultures s'enregistreront, à savoir les céréales, les légumineuses, la betterave à sucre et les autres cultures. Ces dernières verront leurs parts dans l'assolement globale baisser de $1 \%, 3 \%, 4 \%$ et $1 \%$ respectivement. Au moment où ces cultures accuseront ces baisses, d'autres cultures, deviendraient plus rentables et verront leurs superficies augmenter. Il s'agit des agrumes, des fourrages, de l'olivier et du maraichage qui augmenteront respectivement de $1 \%, 6 \%, 1 \%$ et $6 \%$. 
Tableau 1. Assolement des cultures avant et après simulation et leur pourcentage de variation en ha

\begin{tabular}{|c|c|c|c|}
\hline & SDR $^{\mathbf{6}}$ & SIM1 $^{\mathbf{7}}$ & Variation (\%) \\
\hline Autres & 30478 & 30108 & $-1 \%$ \\
\hline Céréales & 361861 & 359781 & $-1 \%$ \\
\hline Agrumes & 12565 & 12718 & $1 \%$ \\
\hline Fourrages & 41289 & 43821 & $6 \%$ \\
\hline Légumineuses & 24517 & 23739 & $-3 \%$ \\
\hline Maraichage & 10192 & 10839 & $6 \%$ \\
\hline Olivier & 32908 & 33183 & $1 \%$ \\
\hline Betterave à sucre & 10447 & 10069 & $-4 \%$ \\
\hline
\end{tabular}

Les assolements simulés et ventilés selon les différentes zones (Tableau 2) montrent que c'est dans les périmètres de grande hydraulique, irriguant exclusivement à partir des eaux des barrages, où les changements se feront sentir le plus. En effet, dans le périmètre de grande hydraulique des Béni Moussa, les agriculteurs réduiront de façon importante les superficies allouées aux légumineuses pour faire plus de fourrages. La part des superficies dédiées aux légumineuses baisserait de $65 \%$, tandis que celles dédiées aux fourrages, aux maraichages, à la betterave à sucre et à l'olivier augmenteraient d'environ $22 \%, 15 \%, 5 \%$ et $3 \%$ respectivement. Au niveau du périmètre de grande hydraulique des Béni Amir, les parts des céréales et de la betterave à sucre baisseraient de $21 \%$ et $23 \%$ au profit du maraichage et de l'olivier.

Dans le périmètre privé aucun changement ne se ferait sentir malgré l'introduction de cette nouvelle technologie et ce pour plusieurs raisons. D'abord, les agriculteurs ont un accès permanant à une nappe dont le volume est extrêmement important et donc, leurs rendements et leurs performances sont relativement peu sensibles à la technologie et technique d'irrigation adoptées. Ensuite, il s'agit généralement de grandes exploitations agricoles, se trouvant déjà sur la frontière d'efficience et qui jouissent d'une situation optimale avant même l'installation du nouveau système d'irrigation.

${ }^{6} \mathrm{SDR}$ : situation de référence

${ }^{7}$ SIM1 : simulation 
Tableau 2. Assolement des cultures en situation avant et après projet en Ha

\begin{tabular}{|c|c|c|c|c|}
\hline & culture & SDR & SIM1 & Variation (\%) \\
\hline \multirow{7}{*}{ PGH_ABAM } & Autres & 466 & 468 & $0 \%$ \\
\hline & Céréales & 10,591 & 8,405 & $-21 \%$ \\
\hline & Fourrages & 9,220 & 9,085 & $-1 \%$ \\
\hline & Légumineuses & 720 & 744 & $3 \%$ \\
\hline & Maraichage & 1,613 & 1,833 & $14 \%$ \\
\hline & Olivier & 6,943 & 7,021 & $1 \%$ \\
\hline & Betterave à sucre & 2,547 & 1,973 & $-23 \%$ \\
\hline \multirow{8}{*}{ PGH_ABMO } & Autres & 792 & 801 & $1 \%$ \\
\hline & Céréales & 17,315 & 17,421 & $1 \%$ \\
\hline & Agrumes & 7,526 & 7,679 & $2 \%$ \\
\hline & Fourrages & 12,207 & 14,877 & $22 \%$ \\
\hline & Légumineuses & 1,241 & 439 & $-65 \%$ \\
\hline & Maraichage & 2,812 & 3,239 & $15 \%$ \\
\hline & Olivier & 7,583 & 7,780 & $3 \%$ \\
\hline & Betterave à sucre & 4,164 & 4,361 & $5 \%$ \\
\hline \multirow{8}{*}{ PGH_ABMO_PUMP } & Autres & 340 & 340 & $0 \%$ \\
\hline & Céréales & 7,205 & 7,205 & $0 \%$ \\
\hline & Agrumes & 3,249 & 3,249 & $0 \%$ \\
\hline & Fourrages & 5,849 & 5,849 & $0 \%$ \\
\hline & Légumineuses & 495 & 495 & $0 \%$ \\
\hline & Maraichage & 1,261 & 1,261 & $0 \%$ \\
\hline & Olivier & 3,294 & 3,294 & $0 \%$ \\
\hline & Betterave à sucre & 1,688 & 1,688 & $0 \%$ \\
\hline \multirow{7}{*}{ PIR_PV } & Céréales & 17,715 & 17,715 & $0 \%$ \\
\hline & Agrumes & 966 & 966 & $0 \%$ \\
\hline & Fourrages & 3,305 & 3,305 & $0 \%$ \\
\hline & Légumineuses & 15,092 & 15,092 & $0 \%$ \\
\hline & Maraichage & 2,529 & 2,529 & $0 \%$ \\
\hline & Olivier & 1,293 & 1,293 & $0 \%$ \\
\hline & Betterave à sucre & 193 & 193 & $0 \%$ \\
\hline \multirow{8}{*}{ PMH_DIR } & Autres & 781 & 781 & $0 \%$ \\
\hline & Céréales & 2,181 & 2,181 & $0 \%$ \\
\hline & Agrumes & 585 & 585 & $0 \%$ \\
\hline & Fourrages & 1,105 & 1,105 & $0 \%$ \\
\hline & Légumineuses & 393 & 393 & $0 \%$ \\
\hline & Maraichage & 859 & 859 & $0 \%$ \\
\hline & Olivier & 636 & 636 & $0 \%$ \\
\hline & Betterave à sucre & 587 & 587 & $0 \%$ \\
\hline \multirow{8}{*}{ PMH_POM } & Autres & 420 & 420 & $0 \%$ \\
\hline & Céréales & 861 & 861 & $0 \%$ \\
\hline & Agrumes & 240 & 240 & $0 \%$ \\
\hline & Fourrages & 456 & 456 & $0 \%$ \\
\hline & Légumineuses & 158 & 158 & $0 \%$ \\
\hline & Maraichage & 362 & 362 & $0 \%$ \\
\hline & Olivier & 259 & 259 & $0 \%$ \\
\hline & Betterave à sucre & 264 & 264 & $0 \%$ \\
\hline
\end{tabular}




\section{La demande en eau}

Cette section évalue l'impact de la reconversion du système d'irrigation vers l'irrigation localisée sur la balance de l'utilisation de l'eau au niveau de la zone d'étude selon la source de l'eau: eau superficielle, eau souterraine. Ensuite, elle calcule les volumes appliqués par culture et par zone en situation avant et après projet.

\section{Eau superficielle}

Après reconversion du système d'irrigation gravitaire vers le système d'irrigation localisée l'utilisation de l'eau de surface augmenterait globalement de 5\% dans toute la région (Tableau 3). L'intensification de la production dans les périmètres de grande hydraulique serait accompagnée par une augmentation significative des volumes d'eau consommés issus des barrages. Dans ce sens, les deux périmètres de grande hydraulique, irriguant à partir des eaux de surface verront leurs volumes en eau consommés augmenter de $11 \%$, tandis que ceux situés dans les mêmes zones, et ayant recours à la fois aux eaux des barrages et aux eaux souterraines, baisseront significativement leurs consommations en eau de surface d'environ $8 \%$ pour ceux situés dans le périmètre de Béni Amir et $11 \%$ pour les agriculteurs situés dans le périmètre des Béni Moussa. Ces résultats concordent avec les conclusions d'autres études conduites dans le même objectif mais adoptant différentes méthodes. En effet, Benouniche et al. (2014) Ont démontré qu'on utilisant le système d'irrigation goutte à goutte, les agriculteurs appliquent des volumes d'eau d'irrigation supérieur en raison de l'intensification.

Tableau 3. Volumes d'eau de surface annuels destinés à l'agriculture par site de demande en eau avant et après simulation en $1000 \mathrm{~m}^{3}$

\begin{tabular}{|c|c|c|c|}
\hline & SDR & SIM1 & Variation (\%) \\
\hline Grande Hydraulique Beni Amir & 232424 & 257998 & $11 \%$ \\
\hline $\begin{array}{c}\text { Grande Hydraulique Beni Amir } \\
\text { irrigation conjonctive }\end{array}$ & 107830 & 99625 & $-8 \%$ \\
\hline $\begin{array}{c}\text { Grande Hydraulique béni Moussa } \\
\text { Grande Hydraulique béni Moussa } \\
\text { irrigation conjonctive }\end{array}$ & 439497 & 487810 & $11 \%$ \\
\hline Petite et moyenne Hydraulique & 144159 & 128856 & $-11 \%$ \\
\hline irrigation privée & 77160 & 77160 & $0 \%$ \\
\hline Total général & $\mathbf{1 0 3 8 4 6 4}$ & $\mathbf{1 0 8 8 ~ 8 4 2}$ & $0 \%$ \\
\hline
\end{tabular}

\section{Eau souterraine}

Avec la reconversion du système d'irrigation gravitaire vers l'irrigation localisée, l'eau étant localisée au niveau des pieds des plantes, ne s'infiltrera pas pour alimenter la nappe phréatique comme dans le cas du système d'irrigation gravitaire, ce qui affectera négativement les niveaux piézométriques de la nappe. Toutefois, la baisse des infiltrations sera 
accompagnée d'une baisse des volumes apportés, en raison de l'efficience élevé du goutte à goutte, ce qui favorisera l'économie de l'eau au niveau des barrages.

Ainsi on pompera moins d'eau. Comme le montre le Tableau4, on peut dire que le modèle projette des changements importants au niveau des variations des volumes d'eau pompés. Les agriculteurs, pompant l'eau à partir de la nappe phréatique des Béni Moussa, réduiront leurs volumes pompés d'environ $26 \%$. Ainsi, les quantités pompées passeront de 123 millions de $\mathrm{m}^{3}$ à seulement 92 millions de $\mathrm{m}^{3}$. Les agriculteurs situés dans le périmètre des Béni Amir et pratiquant l'irrigation complémentaire à partir de la nappe phréatique se trouvant au-dessous du périmètre, verront leur consommation en eau souterraine baisser de $6 \%$. Les agriculteurs pratiquant l'irrigation privée verront à leur tour leurs quantités d'eau pompées baisser de $4 \%$, ainsi au lieu de pomper au total 258 millions $\mathrm{de}^{3}$, ces agriculteurs pomperont 248 millions de $\mathrm{m}^{3}$.

Tableau 4. Volumes d'eau souterraine destinés à l'agriculture par nappe avant et après simulation en $1000 \mathrm{~m}^{3}$

\begin{tabular}{|c|c|c|c|}
\hline & SDR & SIM1 & Variation (\%) \\
\hline Nappe des Béni Amir & 67812 & 63480 & $-6 \%$ \\
\hline Nappe des Béni Moussa & 123519 & 92019 & $-26 \%$ \\
\hline Nappe du Dir & 11048 & 11048 & $0 \%$ \\
\hline Nappe profonde & 258525 & 248525 & $-4 \%$ \\
\hline Total général & 460904 & 415072 & $-10 \%$ \\
\hline
\end{tabular}

\section{Volumes d'eau appliqués aux cultures}

D'après les projections présentées dans la Figure 2., au niveau des zones de reconversion, les quantités d'eau appliquées aux cultures se réduiront significativement pour toutes les cultures. Par exemple, avec le système d'irrigation gravitaire les céréales consommaient en moyenne $4000 \mathrm{~m}^{3}$ par hectare, alors qu'avec le système d'irrigation localisée, le volume appliqué à cette culture n'est que $2312 \mathrm{~m}^{3}$ par hectare. Le même raisonnement s'applique pour la betterave à sucre, le maraichage, les agrumes et les fourrages, dont les quantités d'eau appliquées par hectare baisseront respectivement de 34\%, $33 \%, 16 \%$ et $9 \%$. Elles passeront de 6548 à $4331 \mathrm{~m}^{3} /$ ha pour la betterave à sucre, de 8465 à $5641 \mathrm{~m}^{3} /$ ha pour le maraichage, de 16081 à $13502 \mathrm{~m}^{3} / \mathrm{ha}$ pour les agrumes et de 12154 à $11007 \mathrm{~m}^{3} / \mathrm{ha}$ pour les fourrages. Cette réduction de consommation en eau s'explique par l'utilisation de l'irrigation localisée, une technologie de pointe dont l'efficience est supérieure à celle de l'irrigation gravitaire. 


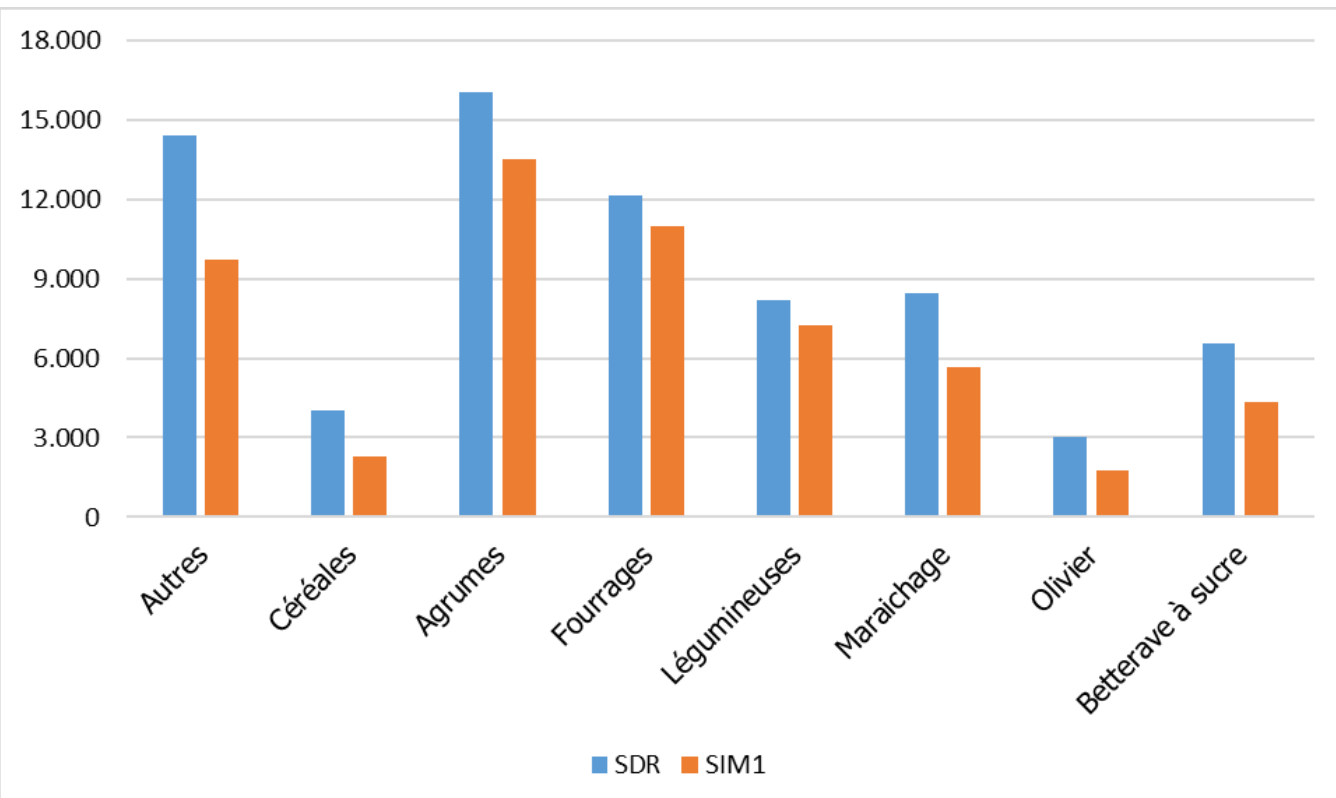

Figure 2. Evolutions des quantités d'eau appliquées aux cultures par hectare avant et après projet de reconversion en $\mathrm{m}^{3} / \mathrm{ha}$

\section{Evolution des rendements}

Sachant que l'irrigation localisée est une technique qui permet, lorsqu'elle est bien mise en œuvre, de contrôler les doses d'eau d'irrigation appliquées aux pieds de chaque plante et les moments d'apport de ces doses, l'équipement des exploitations agricoles par cette technique permettra d'apporter, au bon moment, les doses exactes dont la plante a besoin. Cela garantit l'augmentation des rendements des cultures.

$\mathrm{Au}$ niveau des zones reconverties en système d'irrigation localisée, les rendements des cultures augmenteront significativement par rapport aux rendements obtenus sous système d'irrigation gravitaire. Selon le

Tableau 14, le rendement de toutes les cultures augmentera significativement. La hausse la plus élevée s'enregistrera au niveau de l'olivier, des céréales et des fourrages, qui verront augmenter leur rendement d'environ 13 à 14\%. La plus faible augmentation s'enregistra au niveau des légumineuses qui verront leur rendement augmenter de seulement 3\%. dans le même sens, d'autres études techniques ont montré l'amélioration significative des rendements des cultures pratiquées sous sytème d'irrigation localisée sous condition de controler d'autre variable notamment la salinité (Masmoudi, 2016). 
Tableau 14. Comparaison des rendements réalisés avant et après du système d'irrigation vers l'irrigation localisée pendant une année sèche en $\mathrm{q} / \mathrm{ha}$

\begin{tabular}{|c|c|c|c|}
\hline Cultures & SDR & SIM1 & Variation (\%) \\
\hline Céréales & 41 & 46 & $13 \%$ \\
\hline Betterave à sucre & 503 & 555 & $10 \%$ \\
\hline Oliviers & 30 & 34 & $14 \%$ \\
\hline Agrumes & 31 & 34 & $11 \%$ \\
\hline Fourrages & 493 & 555 & $13 \%$ \\
\hline Légumineuses & 18 & 19 & $3 \%$ \\
\hline Maraichage & 260 & 270 & $4 \%$ \\
\hline Autres & 294 & 308 & $5 \%$ \\
\hline
\end{tabular}

\section{Prix économique de l'eau d'irrigation}

Dans la région de Tadla-Azilal, le prix économique de l'eau moyen s'élève à $0,62 \mathrm{dh} / \mathrm{m}^{3}$ au Béni Moussa, à $0,40 \mathrm{dh} / \mathrm{m}^{3}$ au Béni Amir, et à 0,45 $\mathrm{dh} / \mathrm{m}^{3}$ pour la petite et moyenne et hydraulique. A l'exception du périmètre des Béni Amir, les prix économiques dans les périmètres irrigués dépassent largement les redevances payées par les agriculteurs qui sont de l'ordre de 0,4 $\mathrm{dh} / \mathrm{m}^{3}$ pour les périmètres dominés par les barrages et de $0,16 \mathrm{dh}$ pour les eaux de source.

L'introduction de l'irrigation localisée réduira significativement les prix économiques surtout pour les agriculteurs n'utilisant que l'eau des barrages. Le passage vers un système d'irrigation basé sur la demande en eau, réduira la pression sur les ressources en eau (Figure 3). Toutefois, l'intensification au niveau de la grande hydraulique des Béni Amir se traduira par une demande en eau plus accrue ce qui se reflètera sur le prix économique de l'eau dans la zone. Les agriculteurs pratiquant l'irrigation mixte, à partir des eaux de surface et des eaux souterraines, ne verront pas la valeur économique de l'eau changer car ils ont accès aux nappes souterraines qui représentent une sorte d'assurance en cas de pénurie d'eau. 


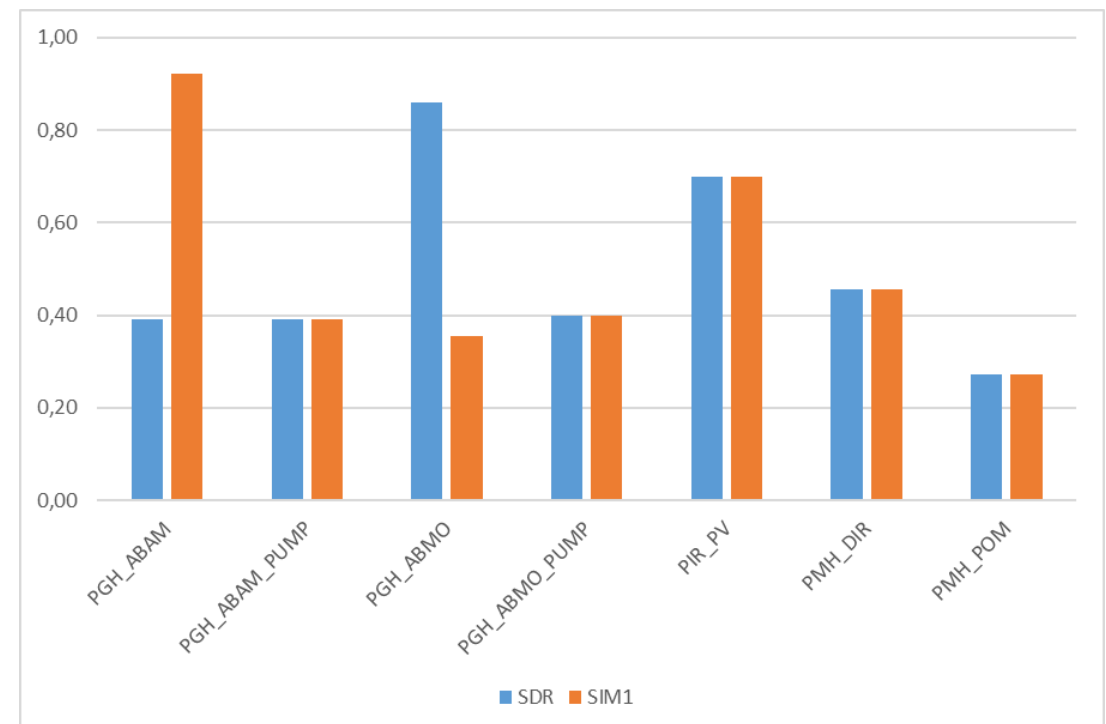

Figure 1. évolution des prix économiques de l'eau d'irrigation avant et après introduction de l'irrigation localisée en dirhams/m3

\section{Evolution de la marge brute agricole}

Après introduction du système d'irrigation localisée, la marge brute totale, issue de la production végétale, passera de 2.81 milliards à 2.86 milliards de dirhams, soit une augmentation de $2 \% \%$. La hausse de revenu la plus élevée s'enregistrera au niveau du périmètre de grande hydraulique des Béni Moussa, pratiquant l'irrigation exclusivement à partir du barrage Bin El Ouidane, qui verra la valeur de la marge brute totale augmenter de $5 \%$. Ainsi, elle passera de 862 millions de dirhams à plus de 902 millions de dirhams. Les agriculteurs situés dans le périmètre de grande hydraulique des Béni Amir verront également leur revenu augmenter de 2\%. Il passera de 341 millions de dirhams à plus de 348 millions de dirhams. Se trouvant déjà sur la frontière d'efficience, les agriculteurs dans les périmètres d'irrigation enregistreront une hausse de revenu très légère, tandis que les autres zones, non concernées par le projet, n'enregistreront aucun changement de revenu. 


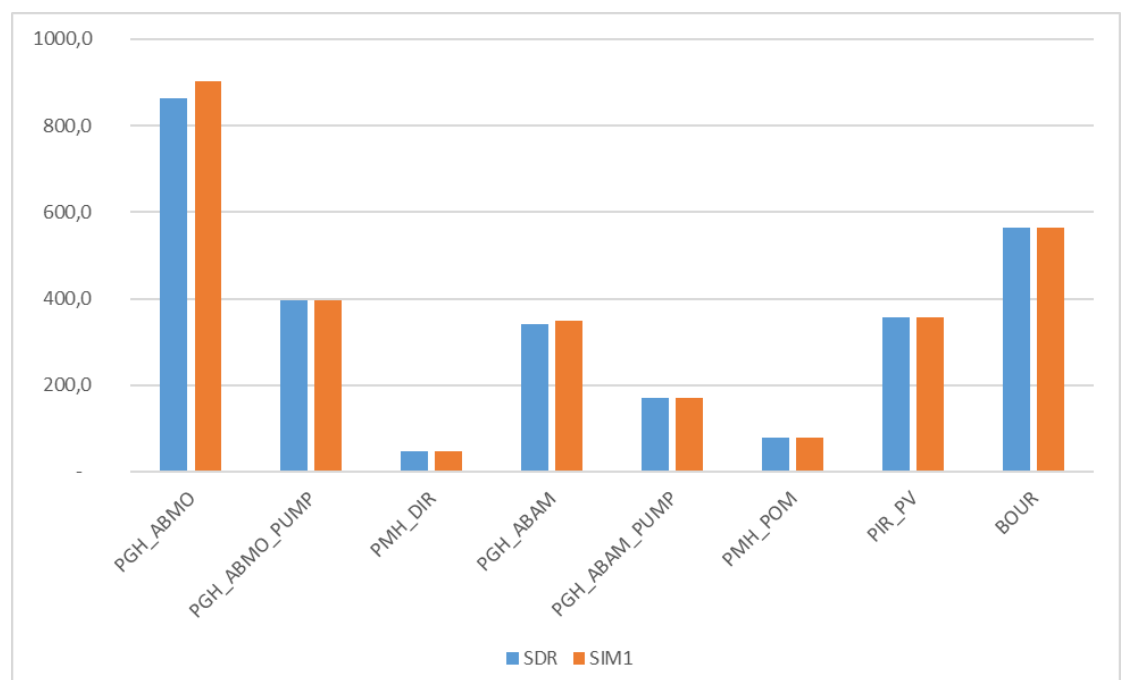

Figure 4. Evolution de la marge brute totale après reconversion du système d'irrigation dans la région de Tadla-Azilal

\section{Conclusion}

L'élaboration du modèle bassin versant dans la zone de Tadla-Azilal, contrairement à ce qui parait, montre que cette zone relativement plus arrosée que d'autres régions au Maroc souffre également d'une rareté d'eau. Ceci se traduit au niveau des résultats par des prix économiques d'eau plus élevés que les prix financiers payés par les agriculteurs dans certains périmètres. En moyenne, le prix économique de l'eau dans la zone s'élève à $0.6 \mathrm{dh} / \mathrm{m} 3$ alors que les redevances payées par les agriculteurs ne dépassent pas $0.4 \mathrm{dh} / \mathrm{m} 3$ dans le cas le plus cher. Par ailleurs, l'introduction du système d'irrigation localisée permettra de réduire la pression sur les ressources hydriques et par conséquent faire baisser le prix économique de l'eau. Selon les résultats du modèle, la reconversion du système d'irrigation du gravitaire vers le goutte à goutte augmentera la consommation en eau issue des barrages en raison de l'intensification, toutefois, ce changement technologique réduira la pression sur les eaux souterraines. Devenue plus chère en raison des frais liés à l'utilisation des goutteurs (frais de filtrage et d'énergie), l'eau souterraine sera de moins en moins utilisée dans le processus de production.

En utilisant cette technologie de pointe capable de répondre aux exigences des plantes en termes d'eau de façon exacte et précise, les agriculteurs augmenteront leur production et amélioreront les rendements des différentes cultures. La hausse des rendements varierait d'une culture à une autre et peut aller jusqu'à $14 \%$, comme c'est le cas pour l'olivier. Certes, l'augmentation de la marge brute agricole totale n'est que de $2 \%$ dans la zone, mais le potentiel est susceptible de dépasser cette valeur. En effet, étant donné que la région de Tadla Azilal est considérée comme l'une des régions les plus 
arrosée au Maroc, et bénéficie de réserves en eau souterraines considérables, les rendements des cultures sont peu sensibles par rapport à l'offre de l'eau sauf en cas d'extrême sécheresse, d'où les performances agricoles importantes qu'enregistre la région même avant l'adoption du système d'irrigation localisée.

\section{References:}

1. Arrifi, E.M. (2009). L'économie et la valorisation de l'eau en irrigation au Maroc: un défi pour l'agriculture irriguée. Symposium international «Agriculture durable en région Méditerranéenne (AGDUMED) ». Rabat. Mai 2009.

2. Belghiti, M. (2004). Valorisation de l'eau et tarification dans les périmètres de " grande hydraulique" au Maroc. Actes du S'eminaire, Modernisation de l'Agriculture Irriguée, Rabat, du 19 au 23 avril 2004.

3. Berger, N. (2010). Modélisation et résolution en programmation par contraintes de problèmes mixtes continu/discret de satisfaction de contraintes et d'optimisation. Modélisation et simulation. Université de Nantes. Français.

4. Benouniche, M., Kuper, M., \& Hammani, A. (2014). Mener le goutte à goutte à l'économie d'eau : ambition réaliste ou poursuite d'une chimère ? Alternatives Rurales, 2, 36-47.

5. Bennouna, A. (2020). Gestion de l'eau au Maroc et changement climatique. Revue Espace Géographique et Société Marocaine. $\mathrm{N}^{\circ} 32$, Février 2020.

6. Bouaziz, A., Belabbes, K. (2002). Efficience productive de l'eau en irrigue au Maroc. Maroc: Revue H.T.E. $\mathrm{N}^{\circ} 124$ - Septembre / Décembre 2002

7. Boukhari, T. R, Naïmi, M., Chikhaoui, M., Raclot, D., Sabir, M. (2019). Evaluation Des Performances Du Modele Agro-Hydrologique SWAT à Reproduire Le fonctionnement Hydrologique Du Bassin Versant Nakhla (Rif occidental, Maroc). European Scientific Journal, ESJ : Vol 15 No 5 (2019): ESJ February EDITION

8. Boularbah, S., Hammani A., BOUARFA, S., Kuper, M. (2017). Analyse de la première expérience de reconversion collective vers l'irrigation localisée: cas du secteur pilote de Tadla. Revue marocaine des sciences agronomiques et vétérinaire 5 (1):76-.82p .

9. Cai, X. (1999). " Modeling framework for sustainable water resources management.' PhD dissertation, Univ. of Texas at Austin, Tex

10. Cai, X., Ringler, C., \& Rosegrant, M. W. (2006). Modeling Water Resources Management at the Basin Level: Methodology and 
Application to the Maipo River Basin. Washington, D.C. : International Food Policy Research Institute.

11. CESE (2014). la gouvernance par la gestion intégrée des ressources en eau au Maroc : levier fondamental de développement durable. Maroc: conseil économique, social et environnemental.

12. CIA (1969). Dahir n 1-69-25 du 10 joumada I 1389 (25 juillet 1969) formant code des investissements agricoles. article 16. Maroc.

13. Dhungel, R., Fritz F. (2016). "Water Balance to Recharge Calculation: Implications for Watershed Management Using Systems Dynamics Approach" Hydrology 3, no. 1 1: 13. https://doi.org/10.3390/hydrology3010013

14. FAO (2012. Crop yield response to water. FAO irrigation and drainage paper 66. food and agriculture organization of the united nations. ISSN 0254-5284

15. Global Water Partnership (2009). Manuel De Gestion Intégrée Des Ressources En Eau Par Bassin. Ministère français des affaires étrangères et européennes. ISBN : 978-91-85321-73-5

16. Global Water Partnership (2012). La gestion de la demande en eau: l'expérience méditérranéenne. Analyse technique. ISBN: 978-9185321-89-6

17. Güntner A., Krol S.M., Araújo J.C, Bronstert A. (2004). Simple water balance modelling of surface reservoir systems in a large data-scarce semiarid region / Modélisation simple du bilan hydrologique de systèmes de réservoirs de surface dans une grande région semi-aride pauvre en données, Hydrological Sciences Journal, 49:5, -918, DOI: 10.1623/hysj.49.5.901.55139

18. Hanks, R. J. (1974). "Model for predicting plant growth as influenced by evapotranspiration and soil water". Agronomy Journal 66:660$665 \mathrm{p}$.

19. Heidecke C. et Kuhn A. (2006). The integrated model of the Drâa valey (le Modèle Intégré de la Vallée du Draa (MIVAD) - Documentation technique. Université de Bonn.

20. Howitt, R. E. (1995. Positive Mathematical Programmi. American Journal of Agricultural Economics, 329-342.

21. MAPMDREF (2021). Statistiques de la production vegetale campagne 2014 / 2015. Ministère de l'agriculture, de la pêche maritime, du développement rural et des eaux et forêts. Rubrique statistiques et veilles.

22. Masmoudi, A., Chargui, Y. (2016). Maîtrise de la salinité sous irrigation goutte à goutte sur une culture de tomate sous serre. Actes du workshop international : gestion intégrée et durable des territoires oasiens. Maroc 
23. Mengoub, F. E., Doukkali, M.R., Lejars, C. (2014). Évaluation de l'Impact économique de la Politique de Reconversion des Systèmes d'Irrigation Gravitaire en Systèmes d'Irrigation Localisée : Cas du Périmètre Irrigué des Béni-Moussa/Tadla. Rabat: Les Economies d'Eau dans les Systèmes Irrigués : Retours d'Expériences au Maroc et à l'international, 20 et 21 octobre 2014, Rabat.

24. Molle F., Tanouti O., Faysse N. (2019). Morocco. In: Molle F., Sanchis-Ibor C., Avellà-Reus L. (eds) Irrigation in the Mediterranean. Global Issues in Water Policy, vol 22. Springer, Cham. https://doi.org/10.1007/978-3-030-03698-0_3

25. Paris, Q., \& Arfini, F. (1996). A positive mathematical programming model for regional analysis of agricultural policies . The regional dimension in agricultural economics and policies, Ancona.

26. Serghini, M. (2003). La tarification des services de l'eau au Maroc. NEW MEDIT, 5-13p.

27. Stella J.M, (2019). Mathematical methodology to calculate the rate of water storage. Int $\mathbf{J}$ Hydro. 3(1):11-16. DOI: $10.15406 / \mathrm{ijh} .2019 .03 .00156$

28. Tsur, Y., Roe, T., Doukkali, M.R, Dinar, A. (2004). Pricing irrigation water: Principles and cases from developing countries. Resources for the Future. https://doi.org/10.4324/9781936331635

29. Vining, K.C., and Vecchia, A.V. (2007). Water-balance simulations of runoff and reservoir storage for the Upper Helmand watershed and Kajakai Reservoir, central Afghanistan: U.S. Geological Survey Scientific Investigations Report 2007-5148, 16p.

30. Zargham M., Mostafa, S., Faghih, A. (2014). Assessment of groundwater recharge in a semi-arid groundwater system using water balance equation, southern Iran. Journal of African Earth Sciences. 95. 